\title{
Termes d'adresse dans la société contemporaine: deux cultures à l'aune du regard du chercheur
}

Danut-Grigore Gavris*

Université de Paris 8, Vincennes-St-Denis, Laboratoire Structures formelles du langage (SFL)

Received: April 2020; Accepted: May 2020

Résumé: Cette étude se propose d'analyser les dérivés du terme frère dans la culture française dont le correspondant est frérot et son équivalent fratè dans la culture italienne. Nous essayerons de comprendre en quoi et comment les deux dérivés représentent ou non les mêmes réalités dans les deux langues, si nous assistons à la même extension sémantique du terme et de quelle (s) manière (s) les locuteurs se servent de ces dérivés dans leurs conversations. L'étude se propose d'être contrastive avec l'objectif de définir les convergences et les divergences de l'emploi des dérivés fratè et frérot dans les langues en question, à savoir l'italien et le français

Mot-clé: termes d'adresse, étude contrastive, frérot vs fratè, culture, sémantique

Abstract: This article's purpose is to analyse the derived forms of term frère in French culture, where its correspondent is frérot, and in Italian culture, where the correspondent is fratè. We will try to show how and why, if yes or not, the two terms represent the same realities in both languages; if there is a same semantic extension and how the speakers of both languages use the two derived forms in their conversations. This study is contrastive, and its objective is also to define convergences and divergences of the use of the two forms fratè and frérot in the languages under analysis, that is to say French and Italian.

Keywords: forms of address, contrastive study, frérot vs fratè, culture, semantics.

\section{INTRODUCTION}

Les recherches en biologie, depuis les soupçons de Buffon', les théories de Lamarck et les démonstrations de Darwin, ont toutes montré une certaine parenté entre les êtres humains, descendus d'ancêtres très simples. Toutefois, les liens de parenté stricto sensu restent délimités à des personnes qui ont des liens de sang. La religion, quant à elle, considère tous les êtres humains comme des frères et des sœurs, fils d'un seul père, Dieu.

Qu'on veuille analyser la question du point de vue biologique, ethnologique ou théologal, il ne nous reste qu'à nous considérer comme une grande famille. Dans cette étude, nous prendrons en considération les dimensions ethnographiques qui impliquent cette question pour étudier un phénomène d'une grande ampleur parmi les jeunes et les moins jeunes de la société contemporaine. S'appeler frère, entre garçons ou filles peu importe, est devenu très à la mode au XXlème siècle. En employant ce terme, ces locuteurs se détachent, petit à petit, de la signification que ce mot a dans une situation où des personnes partagent des ancêtres, pour arriver à une sphère sociale très forte et très importante pour les jeunes, surtout au moment de leur adolescence.

Notre étude vise à analyser l'emploi du mot frère, plus précisément de son dérivé frérot, dans la culture des locuteurs français et des locuteurs italiens de notre époque. Cette démarche contrastive nous permettra de voir de

* Correspondence: Danut-Grigore Gavris: danielegavris@gmail.com

1 Buffon Leclerc, G.-L., (de), 1749. Histoire naturelle. Paris: Imprimerie Royale. 
près les différences d'emploi que ce terme acquiert dans les deux cultures et les nuances qu'il met en évidence. Nous verrons aussi que si pour le français, l'emploi du mot frérot marque une variante de registre de la langue notamment le registre familier, son équivalent italien fratè est employé de façon diatopique. Les deux sont des termes de variétés sociales dus à l'âge des locuteurs qui l'emploient. En effet, on pense généralement que c'est un terme utilisé surtout parmi les jeunes mais les attestations de son emploi montrent que jeunes comme moins jeunes usent et abusent du mot frérot. Toutefois, même si la tranche d'âge est plus large pour l'emploi de frérot par rapport à son équivalent italien dont l'âge d'emploi est plus restreint, il n'est pas utilisé par les personnes au-delà d'un certain âge. Nous verrons cela dans les détails de ce travail. Comme il est question de parentage, nous savons bien que Lévi-Strauss a beaucoup écrit sur le système de parenté. Parmi ses multiples réflexions, celle qui suit a particulièrement retenu notre attention:

Un système de parenté ne consiste pas dans les liens objectifs de filiation ou de consanguinité donnés entre les individus; il n'existe que dans la conscience des hommes, il est un système arbitraire de représentations non le développement spontané d'une situation de fait. (c'est nous qui soulignons)

(Anthropologie structurale, 1958:61)

Cette considération de Lévi-Strauss pourrait nous aider à mieux comprendre l'emploi du terme frère dans une signification moderne. Par «moderne», nous entendons le fait d'utiliser ce terme en dehors de la sphère familiale en dehors des liens du sang qui unissent des semblables partageant, grâce à ces liens, des ancêtres.

Si le système de parenté existe en dehors des liens objectifs de filiation ou de consanguinité considérés par Lévi-Strauss, on pourrait arriver à «justifier » l'usage que l'on fait aujourd'hui d'un terme tellement attaché à la sphère de la parenté de sang. À ce sujet, il suffit d'analyser les familles du XXIème siècle et d'observer que dans les cas d'adoption, les liens de sang sont complètement absents et pourtant ne peut-on appeler cela parenté? II n'est pas de notre compétence d'analyser la notion de "parenté» et encore moins les liens qui la définissent. En tant que linguistes, nous nous intéresserons, dans notre étude, à la dimension que le terme frérot occupe dans la société contemporaine. Certes, pour comprendre le phénomène, il est indispensable de prendre en considération toutes les sciences qui sont impliquées. Le psychologue clinicien Samuel Dock explique qu'utiliser le terme frérot "c'est une manière de se serrer les coudes, d'être ensemble face au monde des responsabilités en cette période difficile de l'adolescence vécue comme une confrontation à la véritable société. Ce bouillonnement tétanisant est ainsi surmonté à travers le groupe $»{ }^{2}$ Pour les jeunes en crise existentielle, trouver une «seconde famille» composée de ses amis auxquels on peut tout dire (ou presque) devient fondamental. Ce qui est étonnant et fascinant à la fois est le fait qu'avec cette extension sociologique, le terme frérot ne perd pas son sens premier. La linguiste Aurore Vincenti explique que "c'est positif, laudatif, c'est un très fort hommage à la notion de fraternité, ça ne fait que célébrer la puissance d'être frère ${ }^{3}$. Ainsi les jeunes tissent des liens qui vont au-delà des notions de consanguinité ou de l'espace familial traditionnel. Ce sont des liens de cœur qui se créent et qui se nouent en accentuant la sensation d'appartenance à un groupe social autre que la famille, groupe qui devient une famille à part entière pour les êtres souvent fragiles que sont les adolescents.

\section{LE CAS DU FRANÇAIS}

Une étude publiée sur le site du journal Le Parisien en juin 2019 constate l'emploi du mot frère parmi les adolescents du quartier des Invalides à Paris; les jeunes se saluent en utilisant le mot frère. Mais il semble que cet emploi soit loin d'être marginal et qu'il ne reste pas limité à ce cadre. Les adultes aussi l'utilisent et en abusent dans leurs conversations. Nous avons constaté que son dérivé frérot est d'usage plus répandu. La morphologie de ce mot montre qu'il est composé du nom frère et du suffixe ot, issu du latin vulgaire (-ottum, -ottam, -ittum, -ittam) qui sert, comme -et, à créer des diminutifs ${ }^{4}$. Le diminutif, nous indique le TLFi, est un «suffixe qui s'ajoute à la base lexicale d'un mot pour souligner la petitesse ou l'insuffisance de l'objet qu'il désigne» mais surtout, en l'attachant à un nom, on obtient une dérivation «à connotation affective ou familière», ce qui semble bien convenir à notre cas. Dans la dérivation du mot frérot nous avons une double connotation qui est affective aussi bien que familière.

Même si son emploi pourrait paraître moderne, ce n'est pas le cas. Le TLFi date l'apparition du mot frérot de 1544 avec la signification de compagnon. II est utilisé par Bonaventure des Periers, dans Joyeux Devis ${ }^{5}$ On l'utilise, en premier lieu, comme terme pour désigner les liens de famille, et ensuite pour désigner le camarade, comme dans le cas de Maupassant6: «Qui est-ce

\footnotetext{
2 Entretien avec Dock, S., in Filles comme garçons, pourquoi nos ados s'appellent tous «frère», de Vincent Mongaillar, LeParisien, 18 juin 2019. [Disponible en ligne à l'adresse: http://www.leparisien.fr/societe/filles-commegarcons-pourquoi-nos-ados-s-appellent-tous-frere-17-06-2019-8095350.php\#xtor=AD-1481423552]

3 Ibidem.

4 Grevisse, M., 1980. Le Bon Usage. Paris: Duculot.

5 CNRTL https://www.cnrtl.fr/etymologie/fr\%C3\%A9rot

6 Maupassant, G., «Les idées du colonel ». In Contes et nouvelles, t. 2, 1884, p. 250
} 
qui prête sa capote? cria Pratique; c'est pour une belle fille, les frérots».

De plus, on nous indique que le terme est aussi un mot de l'argot des prisons, comme dans l'exemple Frérot de la maniche: compagnon de chaîne (France 1907). En ancien français, utilisé au pluriel, le terme signifiait également testicules, comme nous l'indique le dialogue suivant:

[1] La mère: Mais qui te mena espouser?

Le marié: Pourquoy?

La mère: Tu n'as point de fréros.

(Tissier, A., 1986. «Farce de Nouveau Marié ». In Recueil de farces (1450-1550). Genève: Librairie Droz. p. 101.)

Toutefois, dans ce cas le terme comportait l'absence de la lettre $t$ avant le $s$ du pluriel et son emploi au pluriel aujourd'hui ne fait plus référence aux organes masculins. À différence du TLFi, Alain Rey, dans le Dictionnaire historique de la langue française (2010), date l'apparition du mot frérot de 1534 avec la signification de compagnon et le considère comme «un diminutif familier de frère» (Ibid. pag. 5346). Le mot frérot serait concurrencé par frangin, nous fait noter le linguiste. En revanche, le terme frangin serait emprunté au piémontais, un dialecte italien, franzino, «déformation du piémontais fradel, d'après cüzin » (Ibid.), frangin, attesté en 1821 (voir Ansiaume7), au sens de copain, ce qui équivaut à frère.

Néanmoins, toutes ces précisions n'expliquent pas les raisons de l'emploi du mot frérot parmi les jeunes d'aujourd'hui. II semblerait qu'il y ait un lien entre l'utilisation fréquente du terme frérot et celle de l'anglais bro, lui-même diminutif de brother, frère.

Ce serait la culture musicale américaine qui a introduit, avec les chansons hip-hop et rap, le terme dans la société française. En effet, au début les jeunes utilisaient le diminutif de brother, à savoir bro, avant de passer par la suite à frère et à son dérivé frérot. Nous préférons le considérer comme dérivé et non comme diminutif, dans la mesure où, pour les jeunes, leurs camarades ne sont pas plus petits qu'eux mais ils sont des égaux. II n'est pas non plus utilisé pour marquer un sens d'infériorité. Dans ce sens, le terme conserve donc sa dénotation, en même temps qu'il connote l'affectivité ainsi que le sens d'appartenance à une famille ou un groupe social, que les jeunes d'aujourd'hui lui attribuent.

Dans I'Urban Dictionary, on nous informe que brother est:

"one who shares many memories that bring them close to you; the one that shares shows, bands, movies, family, and other interests with you; the person that makes fun of you without it hurting your feelings; the definition of fun and love; one who gives and seeks advice; the one who tells you like it is; the role model; the one who knows how to make you laugh; the one that treats you like you are and nothing more, and nothing less so that you know of your importance, but are humble as well; the one you are most like; someone that generates tears when gone; no matter where you are in the world, you both are still close.» ${ }^{8}$

Nous pouvons constater que c'est une notion qui se détache de la signification sémantique usuelle attribuée à ce substantif pour changer au fur et à mesure de signification. Toutefois, changer de signification ne veut pas dire changer d'affection. Les personnes qui utilisent le mot frérot ont des sentiments tels que ceux qu'ils auraient envers leurs frères consanguins, nous indiquent les attestations et les témoignages.

Le journal Le Parisien nous indique que «l'intrus est apparu dans les années 1980 dans les banlieues, inspiré de la culture hip-hop américaine distillant du «brother» (frère en anglais) et son diminutif «bro» à toutes les sau$\operatorname{ces}^{9} \gg$. Ce substantif serait aussi utilisé par l'ensemble des classes sociales et non pas seulement dans les banlieues. À ce sujet, la linguiste Aurore Vincenti soutient que «cet argot de la dalle se diffuse aujourd'hui de plus en plus vite vers les beaux quartiers grâce notamment au rap, musique démocratique écoutée même dans les milieux les plus aisés ${ }^{10}$. Ce qui reste étonnant est le fait que les filles aussi utilisent le terme frérot pour s'adresser à leurs amies. Une fille résume que «dire frère ou frérot, c'est très masculin. Ma meilleure amie l'utilise quand elle s'énerve. En vrai, c'est pour montrer qu'elle est un peu mec, un peu bad boy ${ }^{11}$. Cependant, on s'aperçoit que la connotation masculine reste attachée au terme quoique des filles l'utilisent. En effet, l'adolescente en question soutient que son amie l'emploie pour ressembler à un vilain garçon, à un macho. Reste le fait que n'ayant pas de preuves concrètes pour attester que le mot frérot est issu de l'emploi de la culture rap, considéré comme dérivée du terme anglais bro, celle-ci est une hypothèse parmi beaucoup d'autres.

Une autre origine du terme frérot pourrait être trouvée dans le terme arabe اخي ('akhi) qui signifie frère. L'immigration vers la France des populations de l'Afrique aurait permis à ce terme d'entrer dans la langue française. En effet, on constate une large utilisation de celui-ci par la population d'Afrique noire, comme l'indique aussi le Cambridge Dictionary pour le cas de brother, lequel est en «US informal sometimes used by a blackman to address or refer to another blackman ${ }^{12}$

\footnotetext{
7 Ansiaume, 1821. Glossaire des mots employés au bagne de Brest. [Disponible à l'adresse: https://www.languefrancaise.net/Source/2033].

8 https://www.urbandictionary.com/define.php?term=brother

9 Ibidem.

10 Ibidem.

11 Ivi: pag. 3

12 Aux États-Unis utilisé par un homme noir pour s'adresser ou faire référence à un autre homme noir.
} 
Le journal Le Parisien ${ }^{13}$ a publié cette image qui introduit quelques treize nuances de frère autour du monde.

Cette image nous aide à comprendre la variété de mots que les jeunes emploient pour s'adresser l'un à l'autre et les liens qui se tissent entre eux. De plus, on voit bien que le mot frérot est strictement lié à la France et s'apparente à d'autres mots comme frolon, frelon et reuf. Nous pouvons observer, sur la droite, les mots venus d'ailleurs.

L'exemple suivant montre que le terme frérot est utilisé avec sa pleine dénotation de compagnon, de quelqu'un qui vise les mêmes objectifs que son locuteur. En effet, c'est sa première dénotation celle de compagnon.

[2] «Afin d'inverser la tendance, Alexandre Lacazette devra se montrer décisif et marquer davantage. En effet, même s'il évoque souvent son entente, à l'intérieur et à l'extérieur du terrain, avec son «frérot» Aubameyang, le numéro 9 ne doit pas se laisser distancer sur le nombre de but.»

(Le Figaro.fr, 17.01.2019) $)^{14}$

On sait tous que l'entente sur le terrain de football est fondamentale pour arriver à emporter le match et les frérots doivent tous faire leur part du travail. Dans l'exemple ci-dessus, le terme frérot est bien dans son contexte. Les joueurs se considèrent tous comme membres d'une grande famille dont l'intérêt commun est de gagner et de remporter les matchs. Le substantif frérot trouve alors sa place et indique les liens familiaux qui se tissent entre les joueurs.

Une autre connotation que nous avons identifiée, grâce à l'emploi du mot frérot, est celle de l'ironie. Analysons l'exemple suivant:

\section{3 nuances de " frère "}

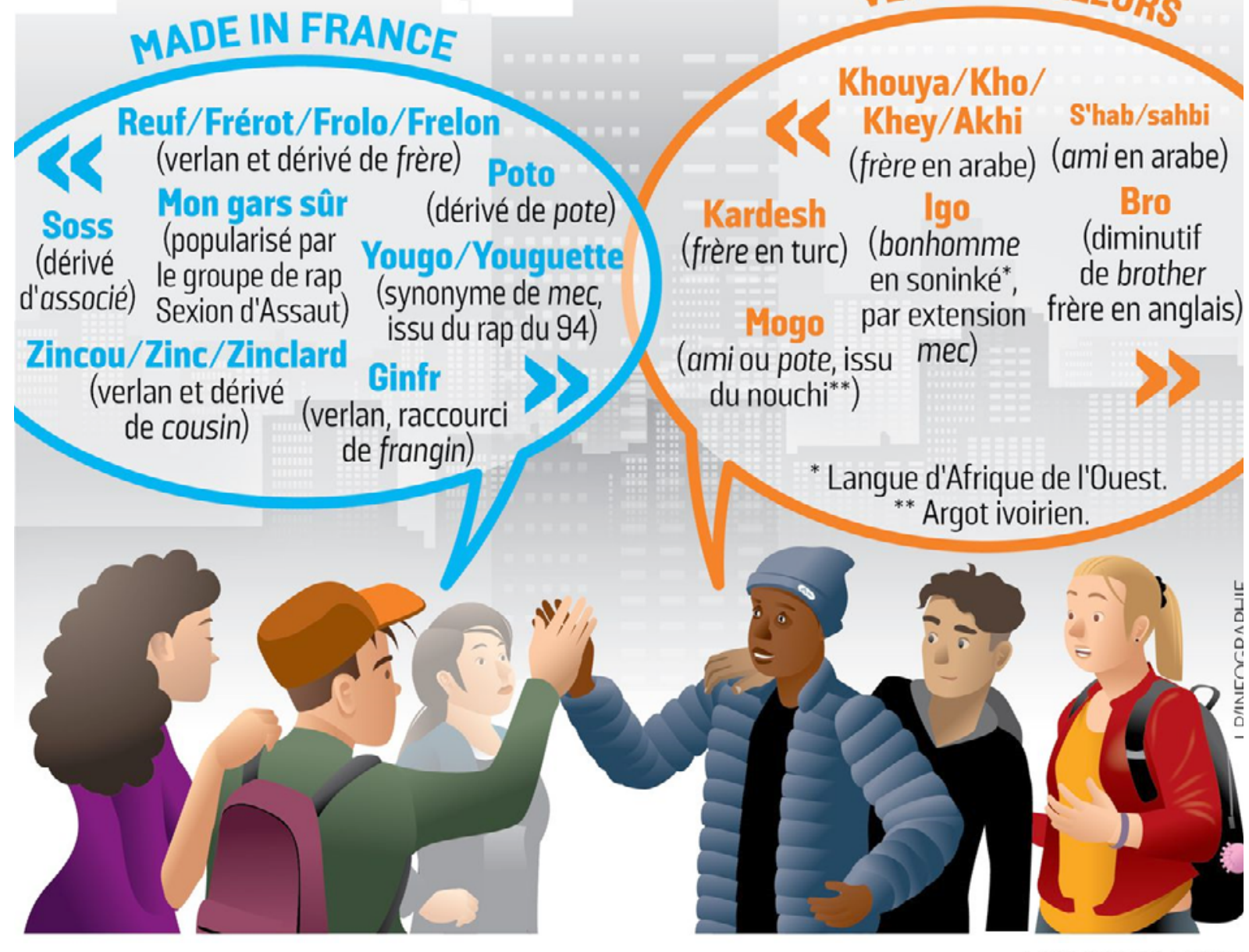

LP/INFOGRAPHIE

13 http://www.leparisien.fr/societe/filles-comme-garcons-pourquoi-nos-ados-s-appellent-tousfrere-17-06-2019-8095350.php

14 http://sport24.lefigaro.fr/football/etranger/angleterre/actualites/lacazette-giroud-des-bleus-bien-pales-avec-lechoc-arsenal-chelsea-941101 
Termes d'adresse dans la société contemporaine: deux cultures à l'aune du regard du chercheur

[3] «Chez certains sportifs aussi, l'ire de Trump a eu du mal à passer. Benjamin Mendy, le latéral de l'équipe de France de football, a donc répondu à sa manière à la provocation. Contraint de déclarer forfait pour les matchs amicaux face aux Pays-Bas et à l'Uruguay, le joueur de Manchester City a dégainé sur les réseaux sociaux mardi en postant un premier message ironique "Don't worry bro» («Ne t'inquiète pas frérot», ndlr). (Le Figaro.fr, 14.11.2018) ${ }^{15}$

Dans ce cas, le diminutif anglais bro est traduit en français avec son diminutif correspondant frérot. Mais son emploi est ironisant. Le joueur ne veut pas considérer, en utilisant ce terme, qu'il a de l'affection à l'égard de Trump, loin de là. Ce que le joueur veut transmettre est son message à Trump de ne plus se mêler des affaires de la France et l'utilisation de deux étoiles, qui symbolisent les titres de champions du monde, accentue la portée ironique de l'énoncé. Dans ce cas, le locuteur viole la maxime de modalité dont la règle est: «soyez clair, bref et méthodique ${ }^{16}$. Le message que le joueur transmet est ambigu, Trump ne peut pas être son ami et l'attaque envers le président des États- Unis est chargée d'ironie. En effet, dans un autre post, sur Twitter, le même auteur assure le président français Macron qu'il s'occupera de Trump, toujours de manière ironique bien sûr.

La forme au pluriel est aussi utilisée par certains pour regrouper un certain nombre d'amis qu'on considère comme des frères. Dans l'exemple ci-dessous Christophe Maé considère ses auteurs-compositeurs comme des frères, en les appelant des frérots.

[4] «Le jury est également Tombé sous le charme de sa chanson éponyme. À l'inverse de Kendji, lui (Christophe Maé) s'est impliqué dans l'écriture et la composition de son titre, épaulé de Michaël Seilham-Ibrahim et de Felipe Saldivia, ses "frérots", comme ils les appellent une fois sur l'estrade. »

(Le Figaro.fr, 17.03.2015) ${ }^{17}$

Si nous avons jusqu'ici abordé le seul cas de l'utilisation orale du terme frérot, la littérature, au moins populaire, n'est pas en reste.

Nous avons trouvé des occurrences de frérot dans le livre Santé ! de Bertrand Sanchez. Voici quelques exemples:

[5]-Super, quel bon vent t'amène Gérard?

-Ton Bordeaux, millésimé pardi, frérot. Clame t'il en se disant qu'il a peut-être gaffé tellement paranoïaque qu'il est, puis continuant sur le ton de la confidence:

- J'ai besoin de ton aide frérot. Si je ne paie pas mes créanciers demain je risque de finir [...].

(Sanchez, B, 2014, Santé!)

Nous pouvons observer, grâce aux marques de l'oral, qu'il est question, dans ce cas aussi, d'un discours oralisé, d'un dialogue entre deux amis. Tout au long du livre nous avons trouvé au moins quatre occurrences du terme frérot.

Un autre exemple remarquable de la littérature est le suivant:

[6] La colère le disputait à la tendresse, les larmes au rire nerveux, l'affection à la trivialité.-Voyons, mon frérot, mon petit gaudebilleux, mon héros, mon escourbette, tu ne vas pas me faire ça à moi! Dis, espèce de branque, mon croquemouche, mon double d'âme, ma trousquette, mon ami de toujours, tu vas revenir, hein?

(Lanzmann, J., 1995. La horde d'or. Paris: Pocket)

Dans ce passage, le chevalier est presque mort et laisse peu d'espérances de vivre. Son ami Grotan lui crie alors sa douleur le voyant près du trépas. L'emploi sémantico-pragmatique du terme frérot trouve ici toute l'affectivité qu'on a pour un frère quand il meurt. Sa signification première est retrouvée également à travers cet emploi.

Comme nous l'avons déjà souligné, l'utilisation de frérot ne reste pas limitée aux adolescents, les adultes l'utilisent aussi. Analysons l'exemple suivant:

[7] Le rappeur de Sevran (Élie Yaffa, connu comme Booba), condamné pour la bagarre avec son concurrent à Orly en août, explique au Parisien qu'il est prêt à «aller jusqu'au bout» et à se battre avec lui dans un match de boxe. "Si tu me proposes un combat, il n'y a pas de souci, frérot», lance-t-il.

(Le Figaro.fr, 16.01.2019) ${ }^{18}$

Dans le cas mentionné ci-dessus, le terme frérot est utilisé pour dénoter une situation négative. II n'a plus la portée affective observée dans l'exemple (2). II est utilisé comme substitut du mot compagnon, dans sa seconde définition donnée par le TLFi. Dans cet exemple, il doit être interprété comme compagnon de combat, de lutte et non pas comme un terme affectif et encore moins comme un sentiment d'appartenance familiale. Cet exemple atteste un mécanisme des modifications

15 http://www.lefigaro.fr/le-scan-sport/2018/11/14/27001-20181114ARTFIG00101-benjamin-mendy-promet-amacron-de-s-occuper-du-cas-de-trump.php

16 Grice H. Paul. 1979. «Logique et conversation». In P. Cole \& J.-L. Morgan (éd.), Syntax and Semantics, vol. 3 Speech Acts, New York: Academic Press, pp. 41-58.

17 http://www.lefigaro.fr/musique/2015/03/17/03006-20150317ARTFIG00353-grand-prix-de-l-unac-christophemae-couronne-par-les-auteurs-compositeurs.php

18 http://www.lefigaro.fr/musique/2019/01/16/03006-20190116ARTFIG00275-kaaris-accepte-le-combat-avecbooba-il-y-aura-un-ring-la-tele-autant-prendre-des-sous.php 
affectives des connotations. II se vérifie en effet une transition de l'affectif au péjoratif, le terme frérot ne renvoie plus à la sphère affective habituelle mais son emploi sémantico-pragmatique devient négatif. Dans la sphère de la proxémie, le substantif frérot se rapproche plutôt des substantifs pair ou encore égal. Si on voulait le remplacer par un autre, on pourrait choisir le diminutif gro, issu du substantif négro, dépourvu de sa connotation négative. Le journal Le Parisien soutient qu'«il est inspiré du terme anglais «nigger» ou «nigga», très populaire aux Etats-Unis au sein de la communauté afro-américaine et que sa variante française «gros» s'est aujourd'hui largement répandue et dépasse sa dimension ethnique». Deux éléments ont attiré ici notre attention: le premier est que le terme frérot n'est pas utilisé seulement par des jeunes adolescents mais par des aussi adultes, et nous reviendrons à ce sujet plus tard. Deuxièmement, le terme est dépourvu de sa connotation affective et prend la place du mot compagnon, ou pair. II est intéressant aussi de prendre en considération les dimensions personnelles du locuteur. En effet, ici on a affaire à un rappeur dont l'origine est sénégalaise. Nous constatons donc que l'emploi du mot frérot n'est pas anodin, nous avons vu tout à l'heure que le mot a vu le jour dans le contexte du rap. Quant à l'origine du rappeur, il n'est pas indispensable de la prendre en considération étant donné le fait que I'utilisation de frérot semble généralisée à l'ensemble des classes sociales.

Un autre exemple atteste de l'emploi du terme frérot à l'interface entre la connotation négative et positive, analysons-le:

[8] Tu es devenu un chantre de la haine, frérot. Tu ne devrais pas plutôt répandre l'amour? Le vrai Love, qui s'arrête pas à la tronche des gens?

(Le Figaro.fr 26 mai 2016) ${ }^{19}$

Cet extrait mérite une explication. En effet, le sémantique liée à l'emploi du terme frérot n'est ni pleinement négative ni pleinement positive. Elle se dessine comme une nuance de la signification d'origine qu'a le terme en question. El Iraki, l'auteur de cet extrait reproche à Jesse Hughes ses propos hors contexte après l'attaque au Bataclan. Highes n'est considéré ici ni en termes d'affection ni en termes d'aversion, mais plutôt comme compagnon, comme semblable.

Nous avons donc pu constater que l'usage du mot frérot ne reste pas limité au cercle des adolescents mais que les adultes l'utilisent aussi. De plus, nous avons observé que la littérature n'est pas dépourvue et que la langue de Molière sait bien intégrer cette nouveauté lexicale. Les attestations de frérot ne restent donc pas confinées à l'oral mais elles sont présentes à l'écrit aussi.
Dans un écrit qui reflète toutefois la langue orale et se caractérise par sa fonction mimétique. On notera, d'ailleurs, que cet emploi hypocoristique du terme frérot n'est pas accompagné de l'article quoique leur connotation ne soit pas archaïque comme l'a fait remarquer Larrivée (2014). En effet, les termes familiaux étaient employés, en 1800 par exemple, seuls et «véritablement sans article en fonction de groupe nominal avec une connotation archaïque» (Larrivée, 2014: 52).

\section{LE CAS DE L'ITALIEN}

Dans cette section, nous analyserons l'emploi du terme fratè, le correspondant du français frérot. Comme le terme français, le mot italien est issu du substantif fratello qui, par apocope, est devenu fratè. L'orthographe et la prononciation de ce terme sont fondamentales pour éviter des confusions avec d'autres de ses homonymes. D'abord, la lettre «e» a un accent grave et contrairement au français, en italien l'accent n'est pas de groupe mais de mot. Si l'accent n'est pas respecté, on pourrait le confondre avec le napolitain frate dont la prononciation est [fr'at] ou [fr'atm] et dont la signification est celle de frère de sang.

Quant à son histoire, le terme semble être un calque de l'anglo-américain brother, comme nous l'avons déjà vu au sujet du français. Nous pouvons considérer deux possibilités pour l'entrée de ce terme dans le langage des jeunes italiens. La première est celle qui concerne les films américains, qui sont transmis à la télévision italienne traduits en italien. Donc, le célèbre bro serait traduit en italien avec fratè. Toutefois, une question se pose tout de suite: pourquoi le terme fratè est-il utilisé seulement dans le dialecte romain?

La seconde option serait celle du rap. En effet, ce serait un calque de la langue du rap dérivé du mot anglais bro. Cette option serait la plus plausible étant donné que dans la banlieue romaine, le rap est la seule culture musicale des jeunes banlieusards. Le terme fratè est utilisé majoritairement dans le dialecte romain, dans la banlieue de RomaEst, à l'Est de Rome. Des attestations sont présentes dans le rap napolitain et l'on constate, avec le temps, une diffusion de l'emploi du terme dans presque toute la zone de Rome. Toutefois, cette constatation reste à vérifier.

II nous semble opportun de faire une remarque par rapport à l'adjectif romain. En effet, il y aurait un autre terme pour qualifier le dialecte de Rome, à savoir l'adjectif romanesco. Comme précisé dans l'article Romano o romanesco? Occorre sciogliere questo primo, sostanziale, dubbio per comprendere davvero se esiste, come e qual è il dialetto della Città Eterna, publié dans le journal italien II Tempo ${ }^{20}$, il y aurait une différence sémantico-pragmatique

19 https://www.lefigaro.fr/musique/2016/05/26/03006-20160526ARTFIG00027-bataclan-un-second-survivantreagit-aux-propos-de-jesse-hugues.php

20 https://www.iltempo.it/roma-capitale/2010/03/07/news/romano-o-romanesco-occorre-sciogliere-questoprimo-sostanziale-dubbio-per-comprendere-davvero-se-esiste-come-e-qual-e-il-dialetto-della-citta-eterna-735921/ 
entre romano et romanesco qui fait que « «romano» e «romanesco» sono stati utilizzati per distinguere le classi sociali capitoline dall'Evo moderno alla caduta dello Stato Pontificio » ${ }^{21}$. On nous informe qu'on utilisait les deux adjectifs pour distinguer les classes sociales de la ville de Rome, et que les termes étaient utilisés du Moyen Âge jusqu'à la chute de l'État pontifical. On définit avec l'adjectif romain la noblesse, la haute et la moyenne bourgeoisie et on utilise l'adjectif romanesco (dont la traduction française n'est pas romanesque) pour identifier le peuple, les artisans, les marins, les boutiquiers, etc. Cependant, nous avons pu remarquer, au cours de notre enquête sur le terrain, que la plus grande partie des habitants de la Ville Éternelle utilise le dialecte dans leur vie quotidienne. À la lumière de ces faits, nous privilégions l'adjectif romain aux fins de notre recherche, mais nous précisons qu'il s'agit d'une question de choix.

Le dialecte romain est assez particulier par rapport aux autres dialectes d'Italie car il semble disparaître à quelques kilomètres de Rome. À partir des bourgs qui entournent la capitale, le dialecte change complètement. La capitale italienne est donc une exception au continuum linguistique ou continuuum dialectal dans lequel on devrait avoir un «gradual change of the language», comme soutenu par Wardhaugh et al. (2015)22. En effet, les linguistes indiquent que «over large distances the dialects at each end of the continuum may well be mutually unintelligible, although speakers can easily understand people in neighbouring area» (Ibid.: 39). Ce qui n'est pas le cas avec le dialecte romain qui se limite strictement à la zone de Rome et à ses banlieues. Une explication possible à l'intelligibilité des Romains avec les autres bourgs, nous expliquent les chercheurs, est qu'à un moment donné les gens habitant Rome ont dû créer un dialecte leur permettant de communiquer avec les visiteurs qui affluaient dans la capitale pour différentes raisons ${ }^{23}$. Le dialecte romain se différencie des autres par ses expressions colorées et non par un lexique trop riche. On a affaire à un dialecte aux expressions multiples plutôt qu'à un dialecte à un vocabulaire très, voire trop varié. Toutefois, ce charme du dialecte romain n'a pas été apprécié par tous. Le premier à le détester, avant qu'il ne soit tel qu'on le connaît aujourd'hui, a été le grand poète italien Dante. Dans le chapitre 9 du premier livre de son De vulgari eloquentia, il s'exprime ainsi:

Sicut ergo Romani se cunctis preponendos existimant, in hac eradicatione sive discerptione non inmerito eos aliis preponamus, protestantes eosdem in nulla vulgaris eloquentie ratione fore tangendos. Dicimus igitur Romanorum non vulgare, sed potius tristiloquium, ytalorum vulgarium omnium esse turpissimum; nec mirum, cum etiam morum habituumque deformitate pre cunctis videantur fetere. Dicunt enim: Messure, quinto dici?24

Dante qualifiait le dialecte romain de discours triste, tristiloquium, ce qui nous fait penser qu'il ne l'appréciait guère. Or, nous ne connaissons pas trop le dialecte romain de l'époque de Dante mais nous connaissons très bien son idée par rapport aux dialectes. En effet, le dialecte qu'il aimait le plus était celui de Toscane. Nous ne nous attarderons pas trop sur la question des dialectes en Italie, qui ne constitue par l'objet de cette étude, mais un bref aperçu nous semblait utile pour comprendre la particularité du dialecte romain.

Un article sur le journal la Repubblica soutient que «sotto i 25 anni dentro il raccordo anulare sono tutti zì e fratè, tutti zii e fratelli » ${ }^{25}$ (en dessous de 25 ans et intra-muros on s'appelle tous oncles, fratè, on est tous des oncles et des frères). Un autre terme que les garçons utilisent et dont nous ne nous occuperons pas dans cette recherche est le terme zì qui signifie oncle, lui aussi issu du dialecte romain. L'article dont nous avons parlé ci-dessus nous indique que la zone où l'on emploie le terme fratè est Rome intra-muros. II confirme que le terme fratè n'est pas utilisé seulement dans la périphérie est de Rome mais aussi dans le centre de la ville. De la même manière que les journalistes français ont entendu le terme frérot aux Invalides, nous l'avons entendu à l'un des plus célèbres lycées de Rome, le lycée Torquato Tasso. Nous pouvons donc affirmer qu'au centre ou moins au centre de la ville de Rome, tous les jeunes garçons utilisent le terme d'appellation fratè avec leurs compagnons.

Avec leur chanson «Ciao fratè ${ }^{26}$, Er Costa et Matt Er Negretto témoignent des emplois à tort et à travers du terme fratè.

\footnotetext{
21 "Romano" et "Romanesco" ont été utilisés pour distinguer les classes sociales de la capitale du Moyen Âge jusqu'à la chute de l'État pontifical. (notre traduction).

22 Wardhaugh, R., Fuller, M.-J., 2015, An introduction to Sociolinguistics, Oxford: Blackwell

23 Frasca, S., 2010, Lessico Romanesco.

24 Étant donné que les Romains pensent devoir se placer avant tous les autres, dans notre procédure de nettoyage ou déracinement, pour les contenter, on les abordera en premier, même si on doit dire qu'ils ne devraient même pas être cités dans aucun discours sur les dialectes vulgaires. En effet, on pourrait dire que le dialecte romanesque n'est pas un dialecte vulgaire, mais un discours triste, c'est le pire de tous les dialectes italiens. Et cela n'est pas étonnant vu le fait qu'ils sont les plus corrompus de tous à cause de leurs monstruosités, de leurs habitudes et de leurs coutumes. Au fait, ils disent: Messure quinto dici? (notre traduction).

25 https://ricerca.repubblica.it/repubblica/archivio/repubblica/2015/06/23/da-blastare-a-bella-zi-il-romanescounder-25Roma01.html?awc=15069_1566403242_02c35ce09153fc154b2836fa40fbda09\&source=AWI_DISPLAY 26 https://www.youtube.com/watch?v=0g6pmCkloql
} 
[9] cè mbotto de gente che me chiama dicendo fratè come stai fratè?

chestaffa' fratè'?

sai che cè?27

Cette strophe nous permet d'entrer directement dans le dialecte romain parce qu'elle est, comme toute la chanson, en dialecte. À partir des noms des musiciens, nous pouvons nous rendre compte que leur provenance est romaine. La particule «er» relève strictement du dialecte romain. Dans ce cas, le mot fratè est utilisé pour s'adresser à son compagnon dans le but de s'informer sur son état général. Comment ça va? est une question assez simple et à la fois neutre mais, en présence du terme fratè, elle acquiert une autre valeur. Come va fratè? pourrait être compris comme «je suis là pour toi mon frérot, dis-moi tout ce qui se passe, tout ce que tu as » et relève de l'intimité de ceux qui l'emploient.

Un autre emploi du terme dans le monde de la musique se retrouve dans l'album du musicien Gemitaiz, dont le titre est «Quello che vi consiglio» (fr. ce que je vous conseille) et dont une chanson est intitulée Fratè.

Nous reportons ici quelques lignes:

\section{[10] Lunedi, fratè}

Resto ancora qui, fratè

Già lo sai, fratè

Mi sveglio così, fratè 28

Nous pouvons constater que chaque vers finit par le terme fratè, ce qui indique sa récursivité tout au long de la chanson. Cet exemple relève de la tendresse qui se déclenche lors de l'utilisation de ce terme. Pour apporter d'autres exemples de son emploi, nous avons analysé la plus grande source d'informations à l'heure du numérique: la page Facebook.

L'exemple suivant propose deux phrases en dialecte romain:

[10] Fratè, esse single è bello na cifra, fai sempre come te pare. Ma buongiorno amore mio 'nse batte! 29 (page Facebook)

Dans ce cas, une jeune fille (dont nous connaissons le profil) déplore le fait qu'être célibataire est très amusant, beau mais un bonjour mon amour n'est comparable à rien. Nous assistons dans ce cas à un emploi décontextualisé du terme; au fait, la fille ne s'adresse pas à quelqu'un mais improvise un dialogue dans lequel le sexe des interlocuteurs ne peut pas être déduit. Ce qui nous fait penser que les filles aussi utilisent le terme fratè.

Un autre exemple d'emploi du terme dans les conversations des jeunes est présent dans l'exemple suivant:

[11] fratè in bocca al lupo per oggi... bella la foto del profilo ${ }^{30}$

(page Facebook)

Dans ce cas, un ami souhaite bonne chance à un autre pour une occasion dont on ne sait pas grand-chose. Dans cet exemple, comme dans celui ci-dessus, le terme est en tête de phrase. Mais les chansons que nous avons analysées révèlent que le terme peut également se trouver en fin de phrase, ce qui indique sa mobilité sur l'axe syntagmatique, sans que cette dernière nuise à la compréhension.

[12] Tantissimi auguroni al nostro bassista Ivan! Bella fratè $^{31}$

(page Facebook)

Dans ce contexte, le terme fratè est employé en fin de syntagme et on comprend que c'est une formule de salutation. En effet, cet emploi est très répandu dans les salutations surtout quand les jeunes se rencontrent. On peut dire que c'est comme dire bonjour, toutefois on ne transmet pas les mêmes sentiments qu'en employant le substantif fratè.

Nous avons passé en revue quelques exemples d'utilisation du mot fratè par les jeunes romains. II faut tout de suite préciser que, qu'au-delà des 25 ans, d'après le journal laRepubblica, le mot fratè n'est plus employé dans la conversation. Le terme reste dans le domaine des jeunes, des très jeunes. Cependant, nous avons trouvé des cas où des personnes d'un âge supérieur à 25 ans ont employé ce terme, mais une étude serait à mener pour approfondir cet aspect des choses.

La littérature populaire romaine semble, elle aussi, marquée par les emplois du mot fratè. Nous avons trouvé quelques exemples que nous avons décidé de prendre en considération et d'analyser.

Dans l'exemple qui suit, nous avons une scène, décrite par l'auteur, qui justifie de l'emploi du terme fratè:

[13]-Bella fratè!... Disse Renato Moncada al suo amico Andrea Montaldo quel pomeriggio di giugno del 1984, quando s'incontrò con il suo amico a P.zza della Chiesa Nuova di Corso Vittorio a Roma.

-Bella fratè!... Si sentì rispondere con un sorriso aperto

27 II y a beaucoup de gens qui m'appellent en me disant fratè, comment tu vas fratè, tu fais quoi fratè, tu sais quoi? (notre traduction)

28 Lundi fratè, je reste encore ici fratè, tu le sais déjà fratè, je me réveille ainsi fratè, (notre traduction).

29 Fratè être célib, c'est un truc d'ouf, tu fais toujours comme tu veux. Mais le «bonjour mon amour» est irremplaçable (notre traduction).

30 Bonne chance pour aujourd'hui, elle est belle ta photo de profil (notre traduction).

31 Bon anniversaire à notre ami bassiste Ivan ! Bella fratè ! (notre traduction). 
Termes d'adresse dans la société contemporaine: deux cultures à l'aune du regard du chercheur

dell'amico Andrea e un "cinque" sonoro assieme ad una pacca sulle spalle. ${ }^{32}$

(Moretta, A.,2013. "dove corri Sara?" (il destino ti raggiungerà!), Roma: Aletti Editore.)

On a le privilège d'assister à un dialogue entre deux amis qui se rencontrent au cœur de Rome. Fratè ici trouve sa justification d'emploi, puisque nous sommes en présence de deux amis qui s'adressent l'un à l'autre. D'autres éléments témoignent de la forte amitié liant les deux amis. Le fait de s'en taper cinq et de se donner une tape sur le dos témoignent de la grande amitié entre les deux interlocuteurs. Dans ce cas, le terme fratè fait partie du groupe nominal et il est employé comme formule de salutation de la part des deux amis.

L'exemple qui suit est en dialecte romain et concerne toujours un dialogue entre deux amis. Dans ce cas, il est diffıcile de comprendre si le terme fratè est utilisé avec son sens affectif.

[14] Castore a Polluce: A fratè, diglie ar vecchietto a cavallo de stà carmo e de nun inarberasse sinnò glie s'arza 'a pressione e ce tocca de fa er mazzo pe' 'mbracallo [...]. . $^{33}$ (Capriotti, L. 2013. Oggi le nuvole ballano tango,

Roma: Ibs.)

Le contexte devient fondamental pour comprendre la fonction du terme fratè utilisé par un des personnages. L'interprétation du signe utilisé ici dépend du contexte d'utilisation de ce signe. Comme rappelé par Jakobson (1963:51) «dans la théorie du langage, depuis le haut Moyen-Âge, on n'a cessé de répéter que le mot, en dehors de son contexte n'a pas de signification». En effet, en analysant le contexte d'énonciation on se rend vite compte que ce n'est pas le même cas présenté dans l'exemple 3 du français. Ici, le mot fratè révèle l'affection qui existe entre les deux amis qui dansent le tango, pendant une fête dans le Campidoglio, et l'un d'entre eux s'adresse à l'autre en utilisant le terme fratè, qu'on peut traduire par cher compagnon, ami, camarade, en l'incitant à expliquer ce qui se passe à la statue de Marc-Aurèle qui s'est inquiété du chaos qu'il y avait dans la place.

Cet exemple est utile pour comprendre deux éléments principaux: d'un côté l'importance du contexte et de sa prise en charge par le chercheur quand nous avons affaire à des interactions spontanées pour donner une interprétation correcte de la situation de communication et, d'un autre côté, la différence d'emploi que nous avons soulignée avec le français.

Nous trouvons souvent ce genre d'emploi dans le dialecte romain parmi les jeunes, où le substantif fratè n'est pas adressé de manière fortuite, mais il indique le degré d'intimité avec ses camarades.

L'exemple suivant explique encore mieux ce que nous venons de dire:

[15] Ohè, fratè, bada che abbiamo solo due croste di pane secco ed una lattina d' acqua benedetta. Qui si muore di fame! ${ }^{34}$

(Uccheddu, R. 2006. Dante avrebbe lasciato perdere. La Riflessione: Cagliari)

Les deux amis se trouvent en difficulté avec la nourriture, l'un s'adresse à l'autre pour attirer son attention sur les denrées alimentaires qu'ils possèdent ne sont pas très nombreuses. Le terme fratè peut être interprété ici autrement que sur le plan de la stricte affectivité. En attirant son attention sur leur peu de nourriture, le premier ami transmet au second le message qu'ils sont tous les deux sur le même bateau et qu'ils risquent de mourir au cas où la nourriture ne suffirait pas. Se partager les dernières miettes de pain relève d'une grande amitié mais aussi de quelque chose qui va au-delà de cette amitié, une dévotion profonde à un ami et ne pas prendre le risque de mourir tous les deux de faim. On peut essayer d'interpréter le mot fratè comme companione $(m)$, forme latine composée de cum «avec» et de panis "pain» attestée dans la Loi Salique (éd. Eckhart, I, 99), qui est à son tour le calque d'un mot germanique du type du gotique gahlaiba «compagnon» c'est-à-dire «celui qui partage le pain avec ${ }^{35}$. Cette terminologie relève du domaine militaire et nous constatons une probable coexistence «à l'origine avec contubernalis "camarade de tente" qui a peut-être favorisé le procédé du calque avec com-initial. ${ }^{36}$

Ces exemples nous ont permis de voir que le terme fratè est fortement utilisé par les jeunes italiens et qu'il transmet l'affectivité qu'il englobe même en dehors des liens de sang. Cette extension sémantique, comme beaucoup d'autres, relève de la capacité des jeunes à tisser des liens autres que familiaux en gardant, tout de même, des sentiments proches de ceux qu'on trouve dans une famille. Le terme fratè n'est pas un simple terme d'adresse, mais plutôt une appellation affective envers une

32 Bella fratè, dit Renato Moncada à son ami Andrea Montaldo cet après-midi-là de juin 1984, quand il rencontra avec son ami à P.zza della Chiesa Nuova du Corso Vittorio à Rome. Bella fratè, s'entendit-il répondre avec un grand sourire par son ami Andrea tout cela accompagné par une tape dans les mains et une tape dans le dos.

(notre traduction)

33 Castore à Polluce: Fratè, dis au vieillard qui chevauche de rester calme et de ne pas s'agiter sinon sa tension va grimper et on devra le bâillonner. (notre traduction).

34 Ohé fratè fais gaffe, on a seulement deux croûtons de pain et une cannette d'eau. On risque de mourir de faim. (notre traduction)

35 Krause, W., 1963. Handbuch des Gotischen. Munich: Beck, pp. 345.

36 CNRTL: https://www.cnrtl.fr/definition/compagnon 
personne qui ne partage pas de liens sanguins avec le locuteur. La sphère de la famille, et nous entendons par famille les membres qui se partagent des liens de sang, élargit ses limites et comme dans l'exemple qui suit, ses appellations à d'autres personnages faisant partie de la vie d'un jeune.

[16] fratè, sorè, a' zia, o' zio... A Napoli siamo tuttiparenti. ${ }^{37}$ (Bernardo, F. 2014, Momenti di insopportabile fastidio Feltrinelli, Milano)

Cet exemple confirme ce que nous avons déjà dit, à savoir qu'à Naples aussi on utilise le terme fratè pour s'adresser à des amis intimes. On voit que les termes réservés aux membres de la famille sont utilisés aussi par les jeunes pour appeler leurs amis. On aurait la coutume d'appeler ses copains frérot, sœur, tante, oncle..., en effet il semblerait qu'à Naples tout le monde soit des proches. Fratè donc se dépouille de ses vêtements familiaux pour prendre les formes d'une affectivité qui change au fur et à mesure avec les amis et selon le degré d'intimité qui évolue.

Cette affectivité fraternelle en amitié peut être retrouvée dans la tradition des relations de la chevalerie féodale. On peut penser ici à la Chanson de Roland, précisément à l'amitié qui liait Roland à Olivier. Deux personnages qui ont servi le roi de France, de la douce France, Charlemagne et qui ont partagé le même sort dans la guerre contre les Sarrasins. Cette amitié entre compagnons très nouée et profonde explique pourquoi au vers 1395 Roland appelle Olivier son frère: Olivier frère... Le mot se répète dans le vers 1456: Olivier, cumpainz, frère mais cette fois-ci le terme frère est anticipé par cumpainz, c'est-à-dire compagnon (dérivé de compainz, compeiz, compaing, companz, compens, compoins, compoingz, compoing, compan, cf. Dictionnaire de l'ancienne langue française et de tous ses dialectes du IXe au XVe siècle, F. Godefroy, 1880-1895, pp. 202).

Roland et Olivier sont unis non seulement par les valeurs qui unissent deux chevaliers qui luttent pour la gloire de leur patrie mais aussi par des sentiments très forts qui unissent deux personnes qui ont des liens de sang, comme dans ce cas de figure Roland et Olivier ${ }^{38}$, même si, précisons-le, tous deux ne sont pas frères. II n'est pas étonnant donc que les jeunes de nos jours aient fait dériver du mot frère le terme frérot et qu'ils l'utilisent dans leurs relations amicales avec leurs semblables.

Ainsi, l'ami devient un alter-ego de la situation, un frère, un frérot, semblable à celui avec lequel on partage des particules de sang, des ancêtres, des signes culturels qui se font propres au groupe auquel on se rattache et duquel on fait partie. Une espèce de conduite d'entraide et de solidarité se met en place, de choses cachées et secrètes, réalités qui sont interdites aux adultes et, par extension, à tous ceux qui ne font pas partie du groupe de fraternité. En revenant à l'exemple 15, les deux amis partagent un sort dur; toutefois, ils gardent leur affection et leur sens d'appartenance de l'un à l'autre et de responsabilité de l'un envers l'autre. Ici entre en jeu le sens du sacrifice, lorsqu'un seul bout de pain reste, il faut que l'un des deux se sacrifie. Le frérot ne représente plus le rival dont le frère s'occupe à la maison avec lequel il se dispute la richesse familiale.

L'exemple de l'italien permet de nous rendre compte du sens attribué au terme fratè dans la communauté banlieusarde romaine. L'emploi exclusif avec ses compagnons et avant un certain âge révèle de la portée non seulement sémantique mais aussi affective des mots en question qui essaie de se détacher des caractéristiques traditionnelles attribuées à fratè. La fraternité relève donc, comme rappelé par Bon (2014), de la culture d'une communauté, se développe et évolue avec elle dans les règles et les limites que celle-ci impose.

\section{CONCLUSIONS}

Décrire des phénomènes linguistiques relève du défı énorme car la société évolue en même temps que nous, les linguistes, la décrivons. Cette étude contrastive nous a permis de nous rendre compte de l'emploi du dérivé du mot frère dans deux cultures assez proches mais éloignées à la fois. En guise de conclusion, nous essayerons de revenir sur les exemples donnés pour étayer les ressemblances et les dissemblances par rapport aux deux cultures tout en gardant un regard critique.

La première chose que nous pouvons remarquer et qui est intéressante, est que les deux dérivés frérot et fratè sont utilisés, en France comme en Italie, dans les quartiers riches comme dans les quartiers pauvres. Les Invalides témoignent de cet emploi pour la région parisienne, et le lycée Torquato Tasso pour ce qui est de Rome. Une question vient alors à l'esprit: est-il vraiment question de culture au sens large du terme? Nous laissons au lecteur la possibilité de répondre.

Un autre élément de ressemblance est l'utilisation des dérivés dans la littérature populaire de chaque pays. Dans la littérature populaire française comme dans celle italienne, les deux mots en question sont employés par les auteurs comme marque d'un code linguistique des adolescents.

Quant à la genèse de l'emploi des termes frérot et fratè, même si nous n'avons pas encore assez d'éléments pour pouvoir conclure sur ce sujet, les intuitions et nos récentes enquêtes du dernier moment nous font penser que dans les deux cultures, les dérivés frérot et fratè sont issus de la tradition rap américaine et ont été introduits à travers cette culture musicale dans les pays en question. Nous rejoignons ce que A. Rey pense quand il dit que «le manifeste poétique qui

37 Fratè, sorè, à zi, ò zio... À Napels on est tous des parents. (notre traduction)

38 Chanson de Roland, édité par Gérard Moignet, Paris: Bordas, 1972. 
soutient cette force d'invention vient s'inscrire entre les lames du rap » ${ }^{39}$. Bien sûr, cette culture musicale a peu de choses en commun avec les immortels de la langue française, et comme dit A. Rey «le rap reste un art oral et oratoire et cette langue se construit de façon à n'avoir jamais à emprunter les couloirs de l'Académie française ${ }^{40}$. Néanmoins, cette idée comporte un inconvénient, faire remonter l'origine de ces emplois au contexte rap implique une limite dans la compréhension du terme en question. Des études plus approfondies dans d'autres langues romanes où l'on utilise le terme frère de la même façon qu'en français et en italien pourraient aider à voir plus clair.

Venons aux divergences culturelles relatives à ce sujet et voyons de près quels sont les aspects contrastifs. D'abord, on peut dire qu'en italien le terme fratè fait partie d'un dialecte romain. Si on prend en considération la définition que la sociolinguiste Romaine donne du mot dialecte, à savoir «has generally been used to refer to a subordinate variety of a language. [...] Dialects of a language tend to differ more from one another the more remote they are from one another geographically » (2000: 2), on devrait arriver à comprendre la variété qui existe entre le dialecte romain et ceux des bourgs autour de Rome. Pour ce qui est de son équivalent français, le terme frérot n'est pas partie intégrante d'un dialecte mais plutôt d'un sociolecte. Romaine (op.cit.) considère que le sociolecte says who we are et affirme donc notre appartenance à une classe sociale qui appartient à son tour à une société. Le terme italien serait alors une variante diatopique tandis que celui français un registre de langue.

Pour continuer dans la direction des divergences, on a pu constater qu'en France, le terme frérot est utilisé même dans des cas d'absence d'affectivité entre les personnes qui l'utilisent (cf. ex. 7 et 8). Tel n'est pas le cas pour l'italien, les exemples que nous avons à disposition nous indiquent que le terme fratè est utilisé si et seulement si les personnes qui l'utilisent ont de l'affection et sont des compagnons.

Un autre point, qui distingue les deux cultures, est la tranche d'âge des personnes qui utilisent les dérivés. En italien, nos exemples nous ont montré que le terme fratè est employé par des personnes de la tranche d'âge de 14 à 25 ans, pas au-delà. Ce qui n'est pas le cas pour le français, où l'on a vu que des adultes tels que Elie Yaffa, par exemple, emploient à tort et à travers le mot frérot.

Ces différences interlinguistiques prises en considération, nous ne pouvons pas nier qu'on assiste aujourd'hui, et cette étude le confirme, à ce qu'A. Rey appelle le langage de génération, qui découd le fil d'or de l'auréole de la langue française, et la foule aux pieds. Ces milieux, comme il les considère, qui sont habités par des êtres humains, agissent sur la langue, la déconstruisent et la construisent à leur guise. Toutefois, il ne faut pas non plus en abuser. II faut maintenir la pureté, l'élégance de la langue française tout en l'enrichissant et en l'adaptant à notre époque.

Cette analyse nous a permis d'étudier aussi les relations qui existent entre des membres qui sont impliqués dans la communication transversale. En effet, on a affaire ici, à ce qu'on appelle en sciences de la communication, à la communication horizontale: les deux membres en interaction ont le même statut. Cependant, il faut prendre en considération le fait que la relation dans l'interaction n'est pas stable «car la relation, c'est là une de ses propriétés majeures, évolue sans cesse au cours d'une interaction» (Kerbrat-Orecchioni, 1992: 63). C'est cette typologie de communication en interaction qui définit des relations (Watzlawick et al.,1972). Non seulement l'émetteur crée la signification, la transmet dans le message et celle-ci est décodée par le récepteur, mais ce dernier crée aussi une signification en intervenant sur le message et c'est dans ce sens que la communication se révèle un comportement exprimant des émotions, des attitudes, des sentiments. C'est ce qui se passe dans le cas où des personnes s'adressant l'une à l'autre s'appellent fratè, ou frérot, selon le contexte de communication. Les deux agissent sur le message en exprimant, à l'aide du mot dérivé de frère, des émotions et des sentiments mais aussi le sens d'appartenance.

Pour conclure, nous pouvons affirmer que la langue est soumise aux changements et aux remaniements de ses locuteurs. Ils sont capables de l'adapter aux contextes variés de la communication humaine révélant ainsi que le langage est une capacité unique qui concerne seulement l'humain.

\section{RÉFÉRENCES}

Alighieri, D., 1304. De vulgari eloquentia.

Ansiaume, 1821. Glossaire des mots employés au bagne de Brest. [Disponible à l'adresse: https://www.languefrancaise.net/Source/2033].

Bon, Norbert. 2014. «Psychanalyse de la fraternité». In Le Journal des psychologues, vol. 315, no. 2, pp. 42-47.

Buffon Leclerc, G.-L., (de), 1749. Histoire naturelle. Paris: Imprimerie Royale.

Devoto, G., Giacomelli, G., 2002. I dialetti delle regioni d'Italia. Milano: Bompiani.

Frasca, S., 2010. Lessico Romanesco. [disponible à l'adresse: http://www.vatrarberesh.it/biblioteca/ ebooks/lessicoromanesco.pdf]

Grevisse, M., 1980. Le Bon Usage. Paris: Duculot.

Grice, H.-P., 1975. «Logic and conversation». In P. Cole \& J.-L. Morgan (éd.), Syntax and Semantics, volume 3: Speech Acts, New York: Academic Press, pp. 41-58. Krause, W., 1963. Handbuch des Gotischen. Munich: Beck, pp. 345.

Jakobson, R., 1963. Essais de linguistique générale. Paris: Les éditons de Minuit.

39 Rey, A., préface à Les mots du bitume de A. Vincenti, 2017.

40 Ibidem. 
Larrivée, P., 2014. «Le groupe nominal épithète. Monsieur le linguiste, Mes amis les linguistes, Les linguistes mes amis ». Linguisticae Investigationes, 27, pp. 47-81.

Orecchioni, C.-K., 1992. Les interactions verbales, tome I, Paris: Armand Colin.

Rey, A.

-2010. Dictionnaire historique de la langue française. Paris: Le Robert.

-2017. préface à Les mots du bitume de A. Vincenti.

Romaine, S., 2000. Language in society. An introduction to Sociolinguistics. Oxford: Oxford University Press.

Simonetti, M., 2015. Slangopedia. Dizionario dei gerghi giovanili. Stampa Alternativa.

Strauss, C.-L., 1958. Anthropologie structurale. Paris: Plon. Vincenti, A., 2017. Les mots du bitume. Paris: Le Robert. Wardhaugh, R., Fuller, M.-J., 2015. An introduction to Sociolinguistics. Oxford: Blackwell.

Watzlawick, P., Helmick-Beavin J. et D. Jackson, 1972. Une logique de la Communication, Paris: Éditions du Seuil.

\section{CORPUS}

Bernardo, F. 2014, Momenti di insopportabile fastidio. Feltrinelli: Milano.

Bertrand Sanchez, Santé!. Lulu.com.

Capriotti, L.2013, Oggi le nuvole ballano tango. Roma: Ibs. Chanson de Roland, édité par Gérard Moignet, Paris: Bordas, 1972

chanson Ciao fratè de Er Costa \& Matt Er Negretto

chanson Quello che vi consiglio de Gemitaiz.

Lanzmann, J., 1995. La horde d'or. Paris: Pocket.

Le Figaro.fr

Maupassant, G., «Les idées du colonel». Contes et nouvelles, t. 2, 1884, p. 250

Moretta, A.,2013. "dove corri Sara?" (il destino ti raggiungerà!). Roma: Aletti Editore.

page Facebook

Tissier, A., 1986. "Farce de Nouveau Marié». In Recueil de farces (1450-1550). Genève: Librairie Droz. p. 101.

Uccheddu, R. 2006, Dante avrebbe lasciato perdere. La Riflessione: Cagliari.

\section{SITOGRAPHIE}

http://temi.repubblica.it/espresso-slangopedia/ category/f-come-fighetto/

http://temi.repubblica.it/espresso-slangopedia/ http://www.turbozaura.it/

https://ricerca.repubblica.it/repubblica/archivio/ repubblica/2015/06/23/da-blastare-a-bella-zi-ilromanesco-under-25Roma0html?awc=15069_156 6403242_02c35ce09153fc154b2836fa40fbda09\& source=AWI_DISPLAY

«Romano o romanesco? Occorre sciogliere questo primo, sostanziale, dubbio per comprendere davvero se esiste, come e qual è il dialetto della Città Eterna». In II TEMPO.it, 7 mars 2010. [Disponible à l'adresse: https://www.iltempo.it/roma-capitale/2010/03/07/ news/romano-o-romanesco-occorre-scioglierequesto-primo-sostanziale-dubbio-per-comprenderedavvero-se-esiste-come-e-qual-e-il-dialetto-dellacitta-eterna-735921/, dernière consultation le 26 septembre 2019].

Entretien avec Dock, S., in Filles comme garçons, pourquoi nos ados s'appellent tous «frère», de Vincent Mongaillar, LeParisien, 18 juin 2019. [Disponible en ligne à l'adresse: http://www.leparisien. $\mathrm{fr} /$ societe/filles-comme-garcons-pourquoi-nosados-s-appellent-tous-frere-17-06-2019-8095350. php\#xtor=AD-1481423552, dernière consultation: 12.05.2020]. 